\title{
Nicotinamide mononucleotide improves energy activity and survival rate in an in vitro model of Parkinson's disease
}

\author{
LEI LU ${ }^{1,2^{*}}, \mathrm{LE} \mathrm{TANG}^{3,4^{*}}, \mathrm{WENSHI} \mathrm{WEI}^{3}, \mathrm{YUNYI} \mathrm{HONG}^{2}, \mathrm{HEYU} \mathrm{CHEN}^{2}$, WEIHAI YING $^{2,4}$ and SHENGDI CHEN $^{4}$ \\ ${ }^{1}$ Department of Neurosurgery, Huashan Hospital, Fudan University, Shanghai 200040; \\ ${ }^{2}$ School of Biomedical Engineering and Med-X Research Institute, Shanghai Jiao Tong University, \\ Shanghai 200030; ${ }^{3}$ Department of Neurology, Huadong Hospital, Fudan University, Shanghai 200040; \\ ${ }^{4}$ Department of Neurology and Institute of Neurology, Ruijin Hospital, School of Medicine, \\ Shanghai Jiao Tong University, Shanghai 200025, P.R. China
}

Received February 17, 2014; Accepted June 17, 2014

DOI: $10.3892 /$ etm.2014.1842

\begin{abstract}
Nicotinamide adenine dinucleotide $\left(\mathrm{NAD}^{+}\right)$repletion has been shown to provide marked neuroprotection from genotoxic agent-induced neuronal and astrocyte cell death. One of the key precursors of $\mathrm{NAD}^{+}$is nicotinamide mononucleotide (NMN). Therefore, it was hypothesized that NMN may attenuate apoptosis and improve energy metabolism in Parkinson's disease (PD)-like behavioral and neuropathological changes, and produce significant beneficial effects. In this study, a cellular model of PD, using rotenone-treated PC12 cells, was established to test the hypothesis that NMN may decrease PD-like pathological changes. Experiments were carried out to investigate cell survival, including an intracellular lactate dehydrogenase (LDH) assay. Apoptotic and necrotic cell death, NAD ${ }^{+}$ levels and ATP levels were also evaluated. It was observed that NMN was able to significantly attenuate the rotenone-induced reduction in the survival rate of $\mathrm{PC} 12$ cells, as assessed by MTT and LDH assays. NMN treatment also significantly reduced the rotenone-induced apoptosis of the cells, as assessed by flow cytometry-based Annexin V/7-aminoactinomycin D staining. Furthermore, NMN restored intracellular levels of $\mathrm{NAD}^{+}$and ATP in the rotenone-treated cells, thus demonstrating the capacity of NMN to ameliorate mitochondrial inhibitor-induced impairments of energy metabolism. The present study indicates
\end{abstract}

Correspondence to: Professor Wenshi Wei, Department of Neurology, Huadong Hospital, Fudan University, 221 West Yan'an Street, Shanghai 200040, P.R. China

E-mail: nihmgh@139.com

Professor Shengdi Chen, Department of Neurology and Institute of Neurology, Ruijin Hospital, Shanghai Jiao Tong University School of Medicine, 2nd Ruijun Street, Shanghai 200025, P.R. China

E-mail: nicemeet@139.com

*Contributed equally

Key words: nicotinamide mononucleotide, Parkinson's disease, energy activity, cell survival that NMN produces significant beneficial effects by attenuating apoptosis and improving energy metabolism in a cellular model of PD. These results suggest that NMN may become a promising therapeutic drug for PD.

\section{Introduction}

A number of studies have demonstrated that nicotinamide adenine dinucleotide $\left(\mathrm{NAD}^{+}\right)$plays an important role in energy metabolism and mitochondrial function, as well as gene expression, calcium homeostasis, aging and cell death (1-3). Multiple studies have also revealed that $\mathrm{NAD}^{+}$is a cytoprotective agent for primary cultures of astrocytes, neurons and myocytes; $\mathrm{NAD}^{+}$treatment has been shown to decrease the necrotic cell death in these types of cells induced by oxidative stress (4), DNA-alkylating agents (5) or oxygen-glucose deprivation (6). A previous study demonstrated that NAD ${ }^{+}$ administration significantly reduced ischemic brain injury in rats (7). One of the key precursors of $\mathrm{NAD}^{+}$is nicotinamide mononucleotide (NMN), which is converted to NAD ${ }^{+}$by nicotinamide mononucleotide adenylyltransferase (2).

Parkinson's disease (PD) is one of most common types of movement disorder. A major pathological change of the disease is the loss of dopaminergic neurons in the substantia nigra pars compacta (8). Although it has been suggested that oxidative stress, mitochondrial dysfunction, inflammation or apoptosis of the dopaminergic neurons in the substantia nigra may play a key role in the pathogenesis of PD (9), the precise mechanisms underlying the pathogenesis of PD are not well understood (10). There has been little effective therapy for treatment of this debilitating disorder (11). Dysfunction of the mitochondria may be involved in the pathogenesis of PD and may become a promising therapeutic target for the disease (12).

In vitro and in vivo studies have revealed that rotenone, a mitochondrial complex I inhibitor, induces PD-like behavioral and neuropathological changes, including the induction of apoptosis and acceleration of $\alpha$-synuclein formation, in PD models $(13,14)$. Since $\mathrm{NAD}^{+}$treatment is able to attenuate the genotoxic agent-induced mitochondrial alterations in neurons and astrocytes (4), it was hypothesized that NMN treatment may attenuate rotenone-induced cytotoxicity. In the current 
study, a cellular model of PD, using rotenone-treated PC12 cells, was established to investigate whether NMN is a protective agent against rotenone-induced cytotoxicity.

\section{Materials and methods}

Cell culture. PC12 cells were purchased from the Cell Resource Center of the Shanghai Institute of Biological Sciences of the Chinese Academy of Sciences (Shanghai, China). The cells were plated at an initial density of $5 \times 10^{4}$ cells/well onto 24-well culture plates to test cell viability, and at a density of $1 \times 10^{6}$ cells/well onto 6-well plates in order to prepare samples for western blot analysis. The culture media used was Dulbecco's modified Eagle's medium containing 4,500 mg/l D-glucose, $584 \mathrm{mg} / \mathrm{l} \mathrm{L}$-glutamine and $110 \mathrm{mg} / \mathrm{l}$ sodium pyruvate (Thermo Scientific, Tewksbury, MA, USA), and also containing $1 \%$ penicillin and streptomycin (Invitrogen Life Technologies, Carlsbad, CA, USA) and $10 \%$ fetal bovine serum (PAA Laboratories $\mathrm{GmbH}$, Linz, Austria). The cells were given $0.5 \mu \mathrm{M}$ rotenone (Sigma, St. Louis, MO, USA) with or without co-treatment with different concentrations of NMN (N3501, Sigma Aldrich, USA). The cells were kept for $24 \mathrm{~h}$ in an incubator with $5 \% \mathrm{CO}_{2}$ at $37^{\circ} \mathrm{C}$. In total, the following four concentrations were tested: $0.1 \mathrm{mM}, 1.0 \mathrm{mM}, 5 \mathrm{mM}$ and $10 \mathrm{mM}$, however, the effects of last three appeared to be similar. Thus, the minimum $(0.1 \mathrm{mM})$ and maximum $(1.0 \mathrm{mM})$ concentrations were selected in order to indicate the effect of improving the energy activity and survival rate of rotenone-treated $\mathrm{PC} 12$ cells/

Determination of cell survival. Cell survival was measured by a quantitative colorimetric assay with 3-(4,5-dimethylthiazol-2-yl)-2,5-diphenyltetrazolium bromide (MTT; Sigma). Following drug treatment, PC12 cells were incubated for $4 \mathrm{~h}$ with $5 \mathrm{mg} / \mathrm{ml}$ MTT. Subsequently the cell cultures were lysed in dimethyl sulfoxide (DMSO; Sigma) for $15 \mathrm{~min}$. The optical absorption at $570 \mathrm{~nm}$ was determined using a plate reader (Synergy2, Biotek, Winooski, VT, USA).

Intracellular lactate dehydrogenase $(\mathrm{LDH})$ assay. Using a previously described method (15), cell survival was quantified by the measurement of LDH activity in cell lysates. Cells were lysed for $20 \mathrm{~min}$ in lysing buffer containing $0.04 \%$ Triton X-100, $2 \mathrm{mM}$ 4-(2-hydroxyethyl)-1-piperazineethanesulfonic acid (HEPES), $0.2 \mathrm{mM}$ dithiothreitol, $0.01 \%$ bovine serum albumin and $0.1 \%$ phenol red. A total of $50 \mu 1$ cell lysates at $\mathrm{pH} 7.5$ were mixed with $150 \mu 1500 \mathrm{mM}$ potassium phosphate buffer (pH 7.5) containing $1.5 \mathrm{mM}$ NADH (Sigma) and $7.5 \mathrm{mM}$ sodium pyruvate (Sigma). The change in the absorbance at $340 \mathrm{~nm}$ (A340 nm) was monitored over $90 \mathrm{sec}$. Percentage cell survival was calculated by standardizing the LDH activity of the sample cell lysates to the LDH activity of the lysates of the control (wash only) cell cultures.

Extracellular LDH assay. LDH is a cytosolic enzyme that is released into the cell media upon cell lysis. Extracellular LDH activity is highly correlated with a major index of cell necrosis: the level of propidium iodide-positive cells (16). Therefore, extracellular LDH activity may be used for assessing the number of cells undergoing cell death (17). In the present study, the extracellular LDH activity of PC12 cells was assessed using a previously described method (16). Briefly, $100 \mu 1$ extracellular media from the samples was mixed with $150 \mu \mathrm{l}$ potassium phosphate buffer (500 mM, pH 7.5) containing $1.5 \mathrm{mM}$ NADH and $7.5 \mathrm{mM}$ sodium pyruvate. Changes in the $\mathrm{A} 340 \mathrm{~nm}$ of the samples was monitored over $90 \mathrm{sec}$ using a plate reader.

Determination of apoptotic and necrotic cell death by flow cytometry-based Annexin V/7-aminoactinomycin D (7-AAD) staining. Following washing twice with phosphate-buffered saline (PBS), the cells were suspended in cold $1 \mathrm{X}$ binding buffer at a concentration of $1 \times 10^{6}$ cells $/ \mathrm{ml}$. A total of $100 \mu \mathrm{l}$ cell suspension was mixed with $10 \mu \mathrm{l}$ Annexin V R-phycoerythrin (PE) conjugate (SouthernBiotech, Birmingham, AL, USA). Following incubation on ice for $15 \mathrm{~min}, 200 \mu \mathrm{l}$ cold $1 \mathrm{X}$ binding buffer was added to each tube, followed by the addition of $10 \mu \mathrm{l}$ 7-AAD (SouthernBiotech). The number of the cells in earlyand late-stage apoptosis, and necrosis, was assessed by a flow cytometer (BD FACSAria II; BD Biosciences, Franklin Lanes, NJ, USA).

Hoechst 33258 staining. The nuclear size of cells was assessed by Hoechst staining (18). Following treatment, cells were fixed in $4 \%$ formaldehyde for $15 \mathrm{~min}$ at room temperature. After washing with PBS, the cells were stained with $20 \mu \mathrm{g} / \mathrm{ml}$ Hoechst 33258 (Sigma) in PBS for 20 min. Images of the stained nuclei were captured under a fluorescence microscope (Leica DMI3000B, Leica, Mannheim, Germany).

Western blot analysis of poly (ADP-ribose) polymerase 1 (PARP-1). Using a previously described method (19), PC12 cells were lysed in radioimmunoprecipitation assay (RIPA) cell lysis buffer [50 mM Tris-HCl, pH 8.0, $150 \mathrm{mM} \mathrm{NaCl}, 1 \% \mathrm{NP}-40,0.5 \%$ sodium deoxycholate and $0.1 \%$ sodium dodecyl sulfate (SDS); Sigma] supplemented with $100 \mu \mathrm{M}$ protease inhibitor cocktail (Roche Diagnostics, Mannheim, Germany). Membrane fractions were separated by centrifugation at $16,000 \mathrm{xg}$ for $20 \mathrm{~min}$ at $4^{\circ} \mathrm{C}$. Protein concentrations were determined using a bicinchoninic acid (BCA) protein assay kit (Thermo Scientific, Rockford, IL, USA). The samples were normalized to $30-\mu \mathrm{g}$ of total protein extract and were fractionated by $10 \%$ SDS-polyacrylamide gel electrophoresis and transferred onto polyvinylidene difluoride membranes using a standard technique. The membranes were blocked with 5\% skimmed milk at $37^{\circ} \mathrm{C}$ for $1 \mathrm{~h}$ and incubated with rabbit PARP-1 (p116/p25) antibodies (1:1,000; Epitomics, Burlingame, CA, USA) at $4^{\circ} \mathrm{C}$ overnight. Horseradish peroxidase (HRP)-linked anti-rabbit immunoglobulin G (IgG; Epitomics) and enhanced chemiluminescence (ECL) substrate (Thermo Scientific) were added and the bands were visualized. The equal loading of samples was confirmed by stripping the membranes and reprobing them with goat polyclonal anti-actin $\operatorname{IgG}(1: 400$, Santa Cruz Biotechnology, Inc., Santa Cruz, CA, USA) and HRP-conjugated rabbit anti-goat IgG (Epitomics). Levels of immunoreactive proteins were determined by densitometric scanning using a ChemiDoc XRS system (Bio-Rad, Hercules, CA, USA).

Determination of the levels of ATP. ATP levels were quantified using an ATP Bioluminescence assay kit HS II (Roche Diagnostics) according to the manufacturers' instructions. Cells were lysed with cell lysis reagent (Roche Diagnostics) 
and $50 \mu 1$ lysates were mixed with $150 \mu 1$ luciferase assay reagent (Roche Diagnostics). The luminescence was detected using a plate reader (Biotek Synergy 2; BioTek Instrument, Inc., Winooski, VT, USA). The protein concentrations of the samples were determined by a BCA assay. The ATP concentrations of the samples were calculated using an ATP standard and normalized against the amount of protein in the samples.

$\mathrm{NAD}^{+}$assay. Following drug treatment, the cells were carefully washed with PBS. The levels of $\mathrm{NAD}^{+}$were measured using a plate reader according to a previously described method (4). Cells were extracted in $0.25 \mathrm{ml} 0.5 \mathrm{~N} \mathrm{HClO}_{4}$, scraped, neutralized with $3 \mathrm{M} \mathrm{KOH}$ and $100 \mathrm{mM}$ sodium phosphate buffer ( $\mathrm{pH}$ 7.0). The levels of $\mathrm{NAD}^{+}$were assessed based on the reduction of MTT to formazan by NADH, which was generated by enzymatic cycling with alcohol dehydrogenase. The rate of optical density (OD) increase at $560 \mathrm{~nm}$ was determined by examining the samples immediately and 20 min after the addition of the sample extracts.

Statistical analyses. All data are presented as mean + standard error. Data were assessed by one-way analysis of variance (ANOVA) followed by the Student-Newman-Keuls post hoc test. $\mathrm{P}<0.05$ was considered to indicate a statistically significant difference.

\section{Results}

Treatment with NMN attenuates rotenone-induced injury of PC12 cells. The present study determined the effect of NMN treatment on rotenone-induced changes in the survival rate of PC12 cells using MTT, intracellular LDH and extracellular $\mathrm{LDH}$ assays. Treatment with rotenone for $24 \mathrm{~h}$ decreased the survival rate of PC12 cells in a concentration-dependent manner, as assessed by MTT assay (Fig. 1A). Since $0.5 \mu \mathrm{M}$ rotenone induced an $\sim 60 \%$ decrease in MTT reduction, this concentration of rotenone was used in subsequent experiments. Co-treatment of the cells with $\mathrm{NMN}(0.1$ or $1 \mathrm{mM})$ and rotenone led to a significantly higher survival rate of the $\mathrm{PC} 12$ cells when compared with the survival rate of the PC12 cells treated with rotenone alone (Fig. 1B). The effect of treatment with NMN alone is shown in Fig.1C.

An intracellularLDH assay was also conducted to determine the effects of NMN and rotenone on cell survival. Treatment of the cells with rotenone caused a concentration-dependent reduction in intracellular LDH activity, which is an index of cell survival (Fig. 2A). This effect was significantly attenuated by NMN co-treatment (Fig. 2B). Cell death was assessed by extracellular LDH assay. It was observed that rotenone concentration-dependently induced the death of the PC12 cells (Fig. 2C), which was significantly attenuated by NMN co-treatment (Fig. 2D).

Treatment with NMN decreases rotenone-induced apoptosis of PC12 cells. Flow cytometry-based Annexin V/7-AAD staining was conducted to determine the early and late apoptosis and necrosis of PC12 cells treated with rotenone and NMN. Phosphatidylserine (PS) is expressed on the outer leaflet of the plasma membranes of early-stage apoptotic cells, which may be stained by labeled Annexin V. Late-stage
A

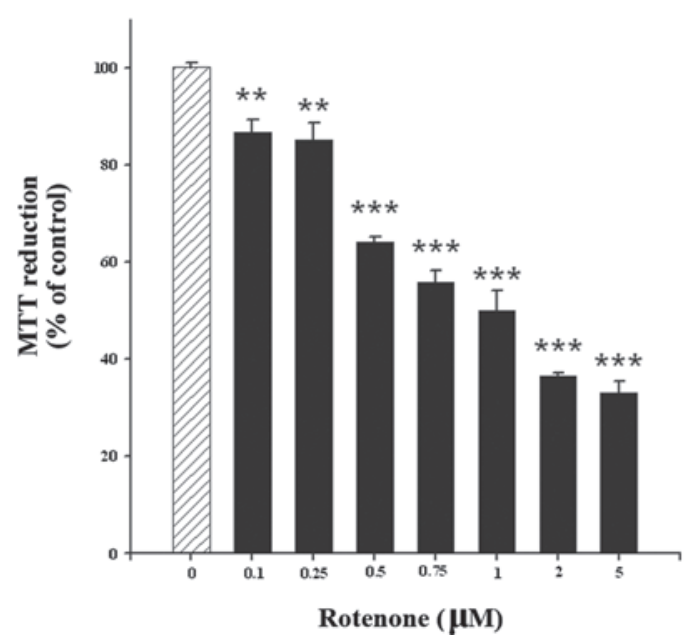

B

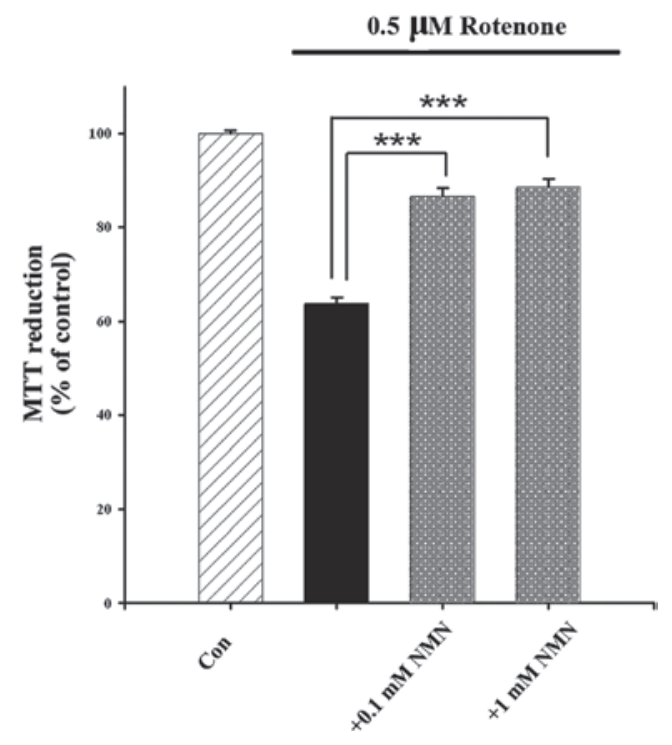

C

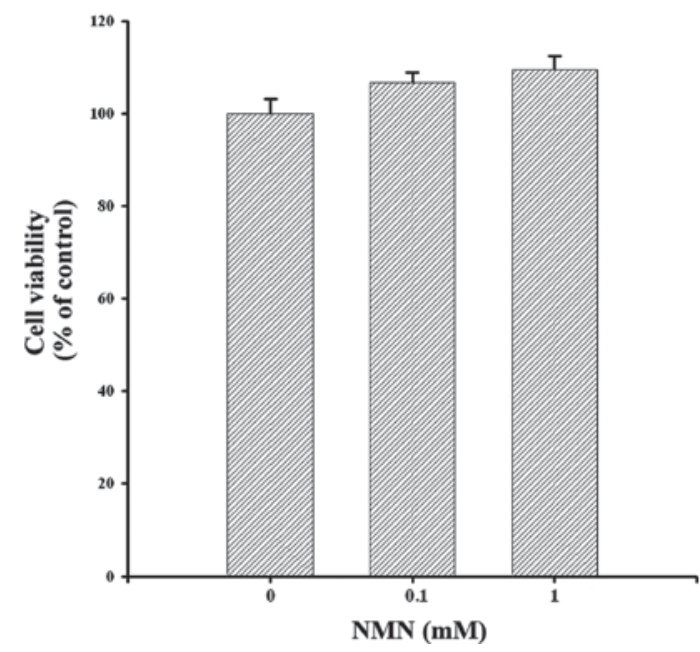

Figure 1. Nicotinamide mononucleotide (NMN) treatment significantly attenuates the rotenone-induced reduction in the survival rate of $\mathrm{PC} 12$ cells. (A) Rotenone concentration-dependently decreased the survival rate of PC12 cells, as assessed by an 3-(4,5-dimethylthiazol-2-yl)-2,5-diphenyltetrazolium bromide (MTT) assay. Following treatment with various concentrations of rotenone for $24 \mathrm{~h}$, the PC12 cell survival rate was determined by MTT assay. (B) NMN treatment significantly attenuated the rotenone-induced reduction in the survival rate of PC12 cells, as assessed by MTT assay; the PC12 cells were co-treated with rotenone and NMN for $24 \mathrm{~h}$. (C) PC12 cells treated with NMN alone. Subsequent cell survival rate was determined by MTT assay. N=12, N, the number of wells for each concentration. Data were collected from $\geq 3$ independent experiments. ${ }^{* *} \mathrm{P}<0.01 ;{ }^{* * * *} \mathrm{P}<0.001$ as indicated, or vs. control (Con). 
A

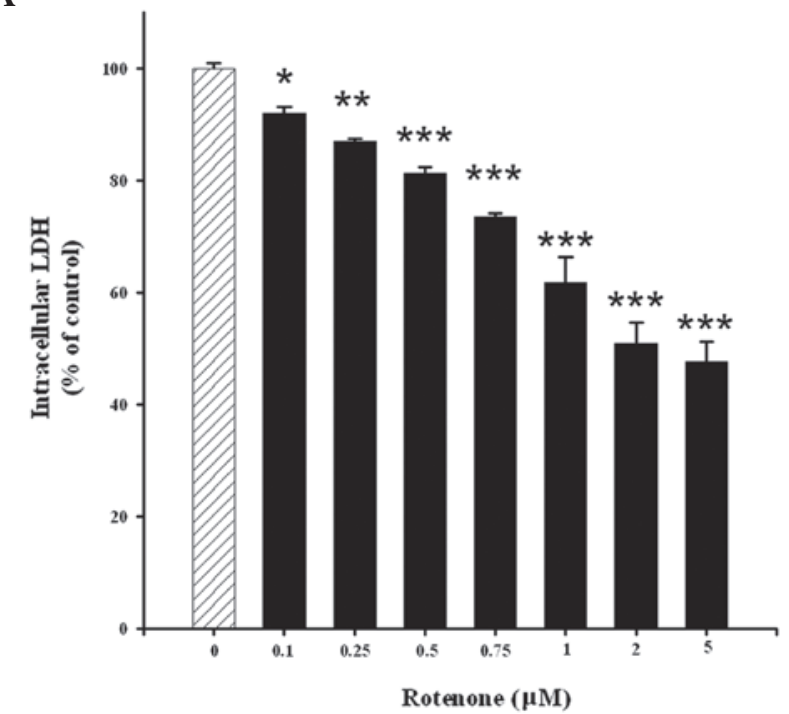

C

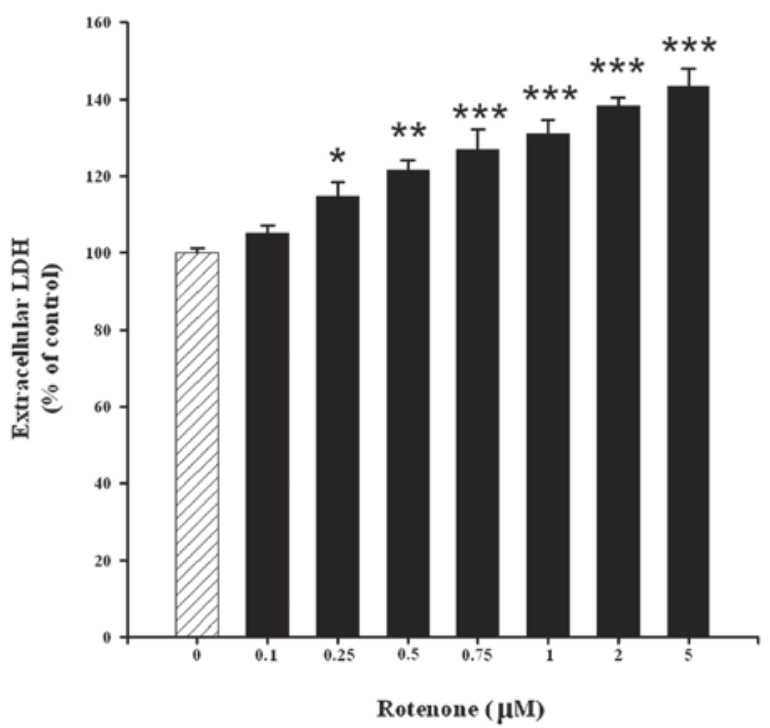

B

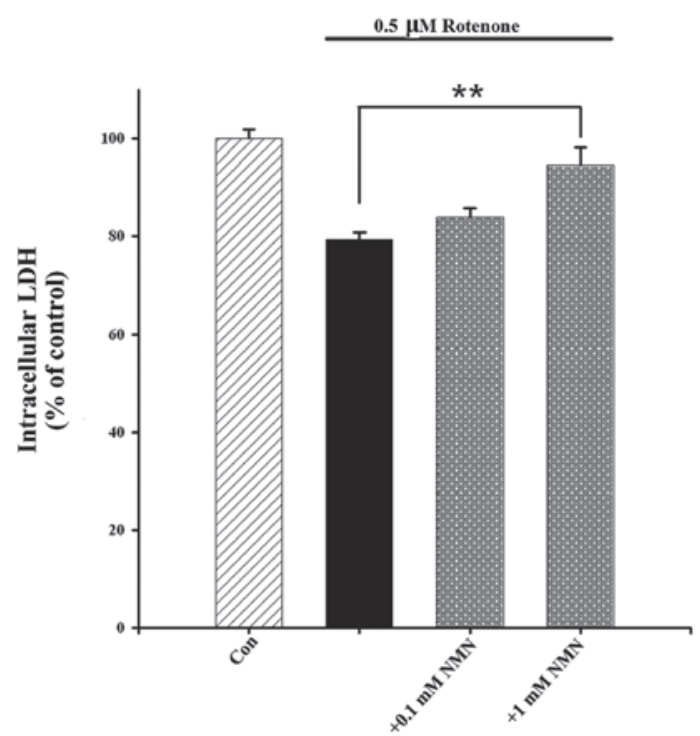

D

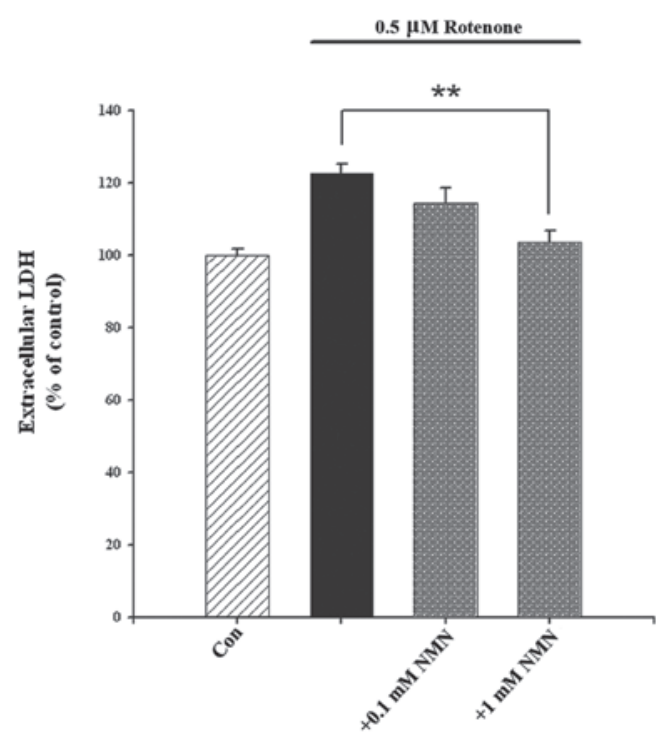

Figure 2. (A) Rotenone concentration-dependently decreased the survival rate of PC12 cells, as assessed by intracellular lactate dehydrogenase (LDH) assay. PC12 cells were treated with various concentrations of rotenone for $24 \mathrm{~h}$. Subsequently, cell survival was determined by intracellular LDH assay. (B) Nicotinamide mononucleotide (NMN) treatment significantly attenuated the rotenone-induced reduction in the survival rate of PC12 cells, as assessed by intracellular LDH assay. (C) Rotenone concentration-dependently induced PC12 cell death, as assessed by extracellular LDH assay. (D) NMN treatment significantly attenuated the rotenone-induced $\mathrm{PC} 12$ cell death, as assessed by extracellular $\mathrm{LDH}$ assay. ${ }^{*} \mathrm{P}<0.05 ;{ }^{* *} \mathrm{P}<0.01 ;{ }^{* * *} \mathrm{P}<0.001$ as indicated or vs. 0 mM rotenone. Con, control.

apoptotic and necrotic cells lose the integrity of their plasma membranes, which become permeable to the fluorescent dye 7-AAD (20). Thus, Annexin V-/7-AAD ${ }^{-}$, Annexin $\mathrm{V}^{+} / 7-\mathrm{AAD}^{-}$, Annexin $\mathrm{V}^{+} / 7-\mathrm{AAD}^{+}$and Annexin $\mathrm{V}^{-} / 7-\mathrm{AAD}^{+}$cells are defined as normal, early-stage apoptotic, late-stage apoptotic and necrotic cells, respectively. Treatment of the cells with $1 \mathrm{mM}$ NMN (Fig. 3A) did not significantly affect the level of cell death compared with that of the control cells (Fig. 3B). Treatment of the cells with $0.5 \mu \mathrm{M}$ rotenone led to a marked increase in the number of Annexin $\mathrm{V}^{+} / 7-\mathrm{AAD}^{-}$cells, as well as increases in the numbers of Annexin $\mathrm{V}^{+} / 7-\mathrm{AAD}^{+}$and Annexin $\mathrm{V}^{-} / 7-\mathrm{AAD}^{+}$cells, compared with the control group (Fig. 3C). Co-treatment of the cells with rotenone and NMN led to significant reductions in the numbers of Annexin $\mathrm{V}^{+} / 7-\mathrm{AAD}^{-}$and Annexin $\mathrm{V}^{+} / 7-\mathrm{AAD}^{+}$cells when compared with the rotenone treatment alone (Fig. 3D). Quantification of these results indicates that NMN significantly decreases the numbers of early- and late-stage apoptotic cells (Fig. 3E).

Nuclear condensation is an indication of cellular apoptosis and may be observed through Hoechst 33258 staining. Furthermore, PARP-1 is cleaved by active caspase- 3 during caspase-3-dependent apoptotic processes. To further determine whether NMN is able to decrease rotenone-induced apoptosis, the levels of nuclear condensation and PARP-1 cleavage in rotenone-treated PC12 cells were assessed. It was observed that rotenone concentration-dependently induced nuclear condensation, and this effect was significantly attenuated by NMN co-treatment (Fig. 4). Western blot analysis also revealed that rotenone concentration-dependently reduced the levels of PARP-1 (Fig. 5A and B). The rotenone-induced reductions in the levels of PARP-1 were significantly attenuated by NMN co-treatment (Fig. 5C and D). 
A
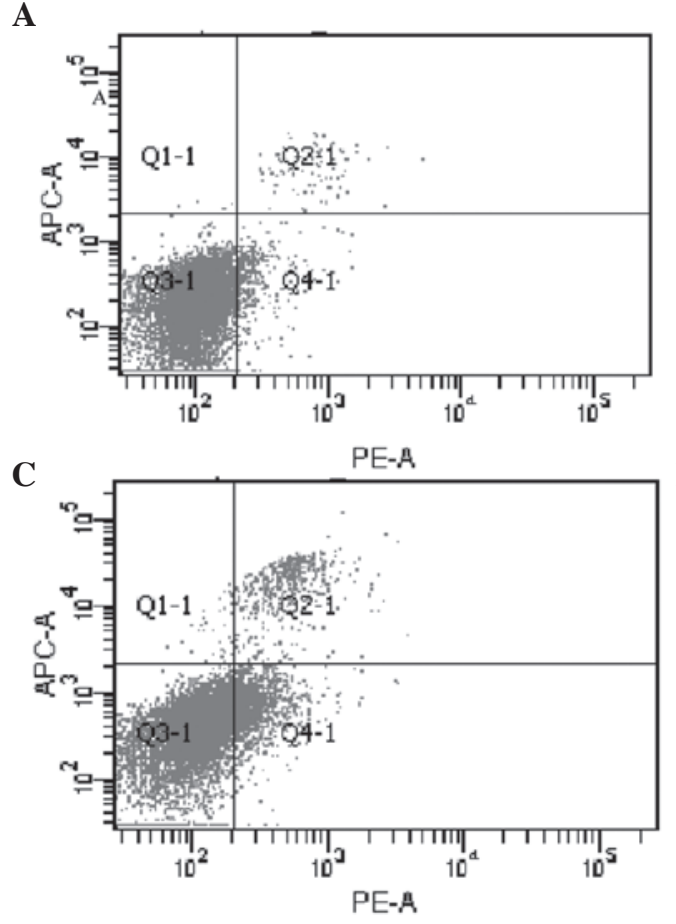

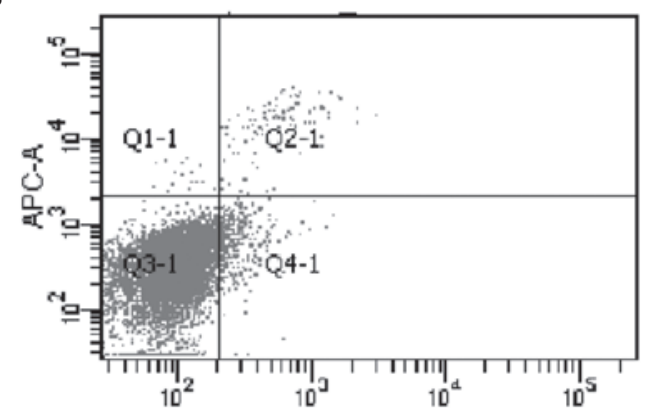

D

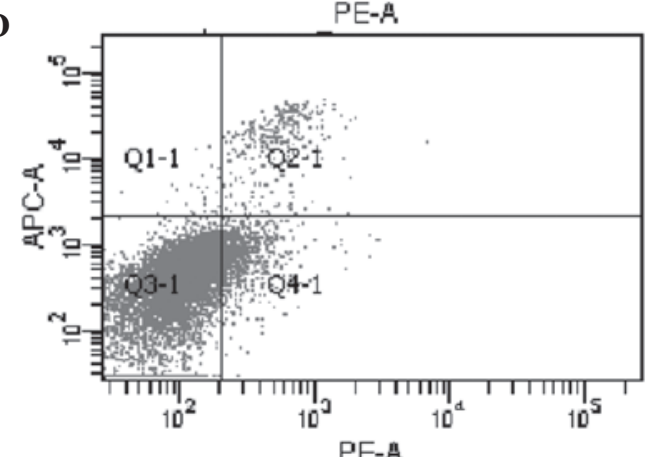

$\mathbf{E}$

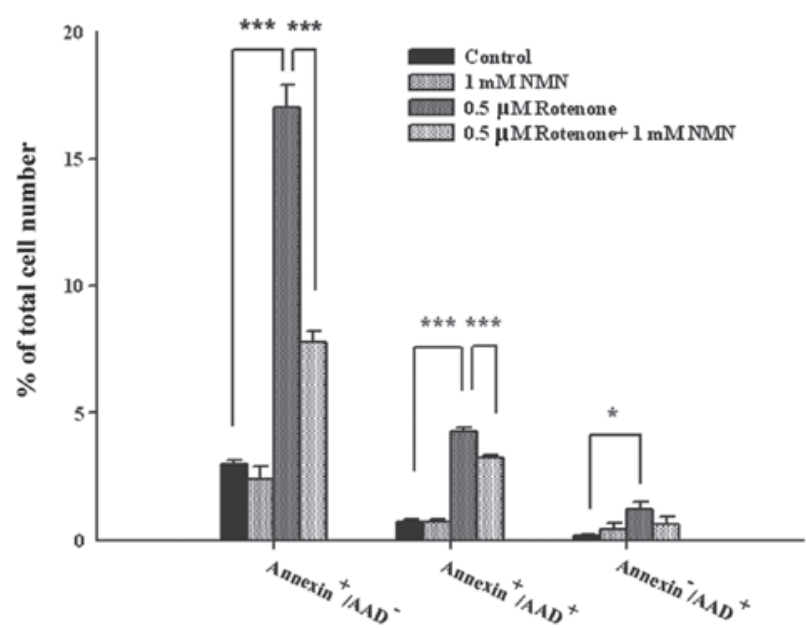

Figure 3. Nicotinamide mononucleotide (NMN) treatment attenuates the rotenone-induced apoptosis of PC12 cells. A flow cytometry-based Annexin V/7-aminoactinomycin D (7-AAD) staining assay was used to determine early- and late-stage apoptosis and necrosis. Treatment of the cells with (A) $1 \mathrm{mM}$ NMN did not significantly affect the level of cell death when compared with (B) the control cells. (C) Treatment of cells with $0.5 \mathrm{mM}$ rotenone led to an increase in the number of Annexin V+/7-AAD- and Annexin V+/7-AAD- cells, which was significantly decreased by (D) the co-treatment of the cells with NMN. (E) Quantification of the FACS results revealed that NMN treatment can significantly decrease apoptosis and necrosis of the rotenone-treated cells among the total cell number. ${ }^{*} \mathrm{P}<0.05 ;{ }^{* * *} \mathrm{P}<0.001$; APC, allophycocyanin; PE, phycoerythrin.

Treatment with NMN restores the intracellular levels of $\mathrm{NAD}^{+}$ in rotenone-treated $\mathrm{PC} 12$ cells. Since NMN is a precursor of $\mathrm{NAD}^{+}$, the present study aimed to determine whether NMN treatment enhances the intracellular levels of $\mathrm{NAD}^{+}$in $\mathrm{PC} 12$ cells. It was observed that treatment of PC12 cells with $0.5 \mathrm{mM}$ rotenone led to a significant reduction in the intracellular levels of $\mathrm{NAD}^{+}$, which was prevented by co-treatment with $1 \mathrm{mM}$ NMN for $24 \mathrm{~h}$ (Fig. 6).

Treatment with NMN attenuates the rotenone-induced reduction in intracellular levels of ATP in PC12 cells. To investigate the mechanisms underlying the protective effect of NMN on rotenone-induced cell death, the effect of NMN treatment on the intracellular levels of ATP in rotenone-treated cells was determined. Rotenone concentration-dependently reduced the intracellular levels of ATP in the cells (Fig. 7A); this effect was significantly attenuated when the cells were co-treated with NMN (Fig. 7B).

\section{Discussion}

The major results of the present study include: i) NMN attenuated the rotenone-induced reduction in the survival rate of PC1 2 cells; ii) NMN reduced the early- and late-stage apoptosis of rotenone-treated $\mathrm{PC} 12$ cells; iii) NMN restored the intracellular levels of $\mathrm{NAD}^{+}$in rotenone-treated $\mathrm{PC} 12$ cells; and iv) the 


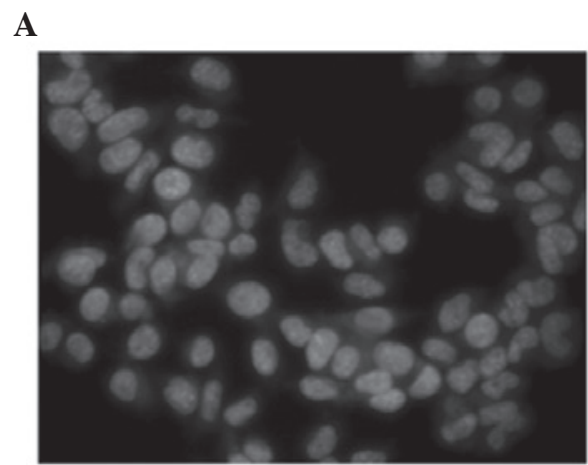

Con

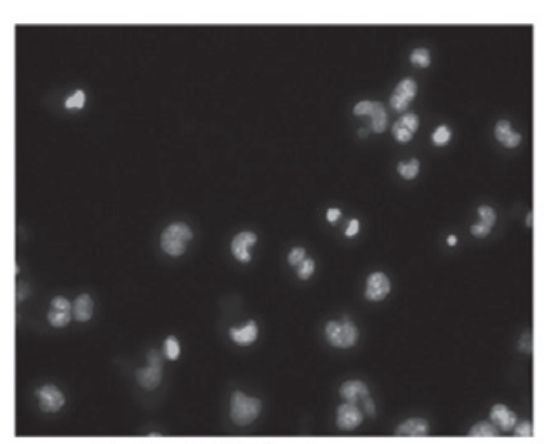

$0.5 \mu$ M Rotenone

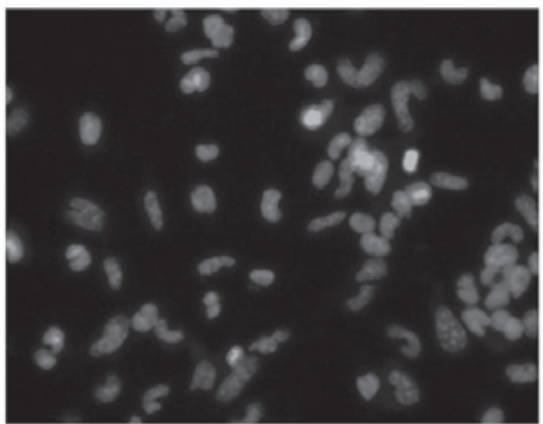

$0.5 \mu M$ Rotenone+1 mM NMN

$\mathbf{B}$

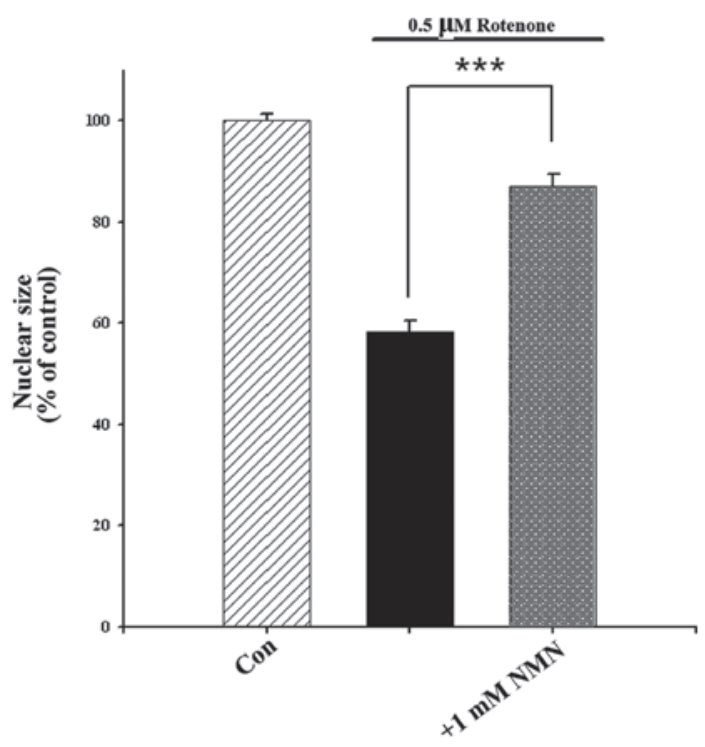

Figure 4. Nicotinamide mononucleotide (NMN) treatment attenuates rotenone-induced nuclear condensation, as assessed by Hoechst 33258 staining. PC12 cells were treated with $0.5 \mu \mathrm{M}$ rotenone, with or without co-treatment with NMN for $24 \mathrm{~h}$. (A) The nuclear condensation of the cells was assessed by Hoechst 33258 staining. The images presented are representative of the those captured in three independent experiments. Magnification, x200. (B) Quantification of the nuclear size of the cells revealed that $\mathrm{NMN}$ treatment significantly attenuated rotenone-induced nuclear condensation. ${ }^{* * *} \mathrm{P}<0.001$.

protective effects of NMN against rotenone-induced cell death were demonstrated through the prevention of rotenone-induced ATP depletion.

The results of the present study suggest that NMN attenuates cell apoptosis and decreases the intracellular levels of ATP in rotenone-treated PC12 cells. Cumulative evidence has suggested that $\mathrm{NAD}^{+}$plays significant roles in a variety of biological processes, including energy metabolism, mitochondrial functions, calcium homeostasis, antioxidation/generation of oxidative stress, gene expression, immunological functions, aging and cell death (2). $\mathrm{NAD}^{+}$treatment has also been found to decrease the rate of apoptosis of primary cultures of neurons, astrocytes and myocytes, induced by various insults (4). NAD ${ }^{+}$acts as a neuroprotective agent via several mechanisms, including the prevention of mitochondrial impairment $(4,21)$, prevention of ATP depletion and glycolytic inhibition $(4,5,21)$, and the enhancement of DNA repair (6).

$\mathrm{NMN}$ is a major precursor of $\mathrm{NAD}^{+}$in the salvage pathway of $\mathrm{NAD}^{+}$synthesis, where it is converted to $\mathrm{NAD}^{+}$in cells by nicotinamide mononucleotide adenylyltransferase (2). The current study demonstrated that NMN treatment was highly protective against the rotenone-induced cytotoxicity of PC12 cells in a cellular model of PD. This was revealed through various cell apoptosis assays, including LDH and MTT assays, and flow cytometry-based Annexin V/7-AAD and Hoechst staining. Furthermore, the present study demonstrated that NMN treatment was able to significantly decrease the rotenone-induced apoptosis of cells, as indicated by experiments that applied flow cytometry-based Annexin V and Hoechst staining, and Western blot analysis of PARP-1.

The mechanisms underlying the protective effect of NMN on the neurotoxicity of rotenone were investigated. The results of the present study demonstrated that treatment with NMN restored the intracellular levels of $\mathrm{NAD}^{+}$and attenuated the reduction in the levels of ATP in rotenone-treated PC12 cells. Since intracellular ATP (22) and $\mathrm{NAD}^{+}(2)$ are mediators of cell survival, the beneficial effects of NMN on the levels of ATP and $\mathrm{NAD}^{+}$may at least partially account for the protective effects of NMN against rotenone-induced cell death. As NAD ${ }^{+}$ restoration may lead to a reduction in the ATP consumption used for $\mathrm{NAD}^{+}$synthesis (2), the $\mathrm{NMN}$-induced restoration of intracellular levels of $\mathrm{NAD}^{+}$may account for the beneficial effects of NMN on the intracellular levels of ATP. 
A

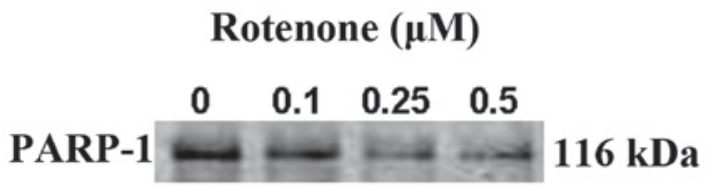

B
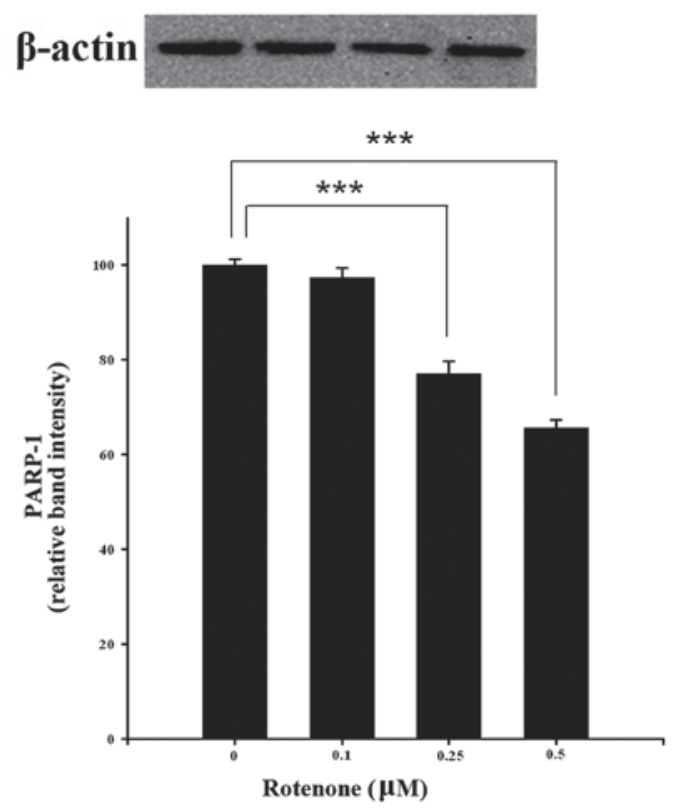

C

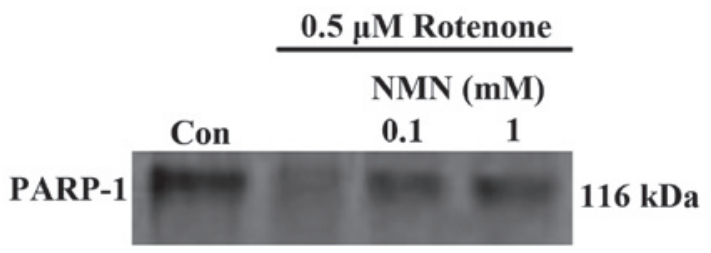

$\beta$-actin

D

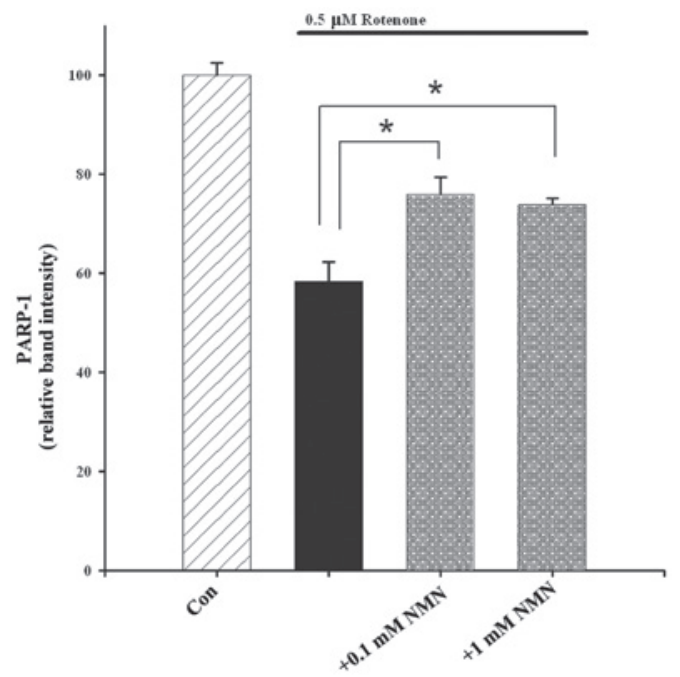

Figure 5. Nicotinamide mononucleotide (NMN) treatment attenuates the rotenone-induced reduction of the levels of poly (ADP-ribose) polymerase 1 (PARP-1) in PC12 cells, as determined by western blot analysis using anti-PARP-1 antibodies. PC12 cells were treated with $0.5 \mu \mathrm{M}$ rotenone, with or without co-treatment with NMN for $24 \mathrm{~h}$. (A and B) $0.5 \mu \mathrm{M}$ rotenone caused a reduction in the levels of PARP-1, as assessed by western blot analysis. (C and D) NMN treatment significantly attenuated the rotenone-induced reduction of the levels of PARP-1. N=9, N, the times of repeats of the experiments. Data were collected from three independent experiments. ${ }^{*} \mathrm{P}<0.05$; Con, control.

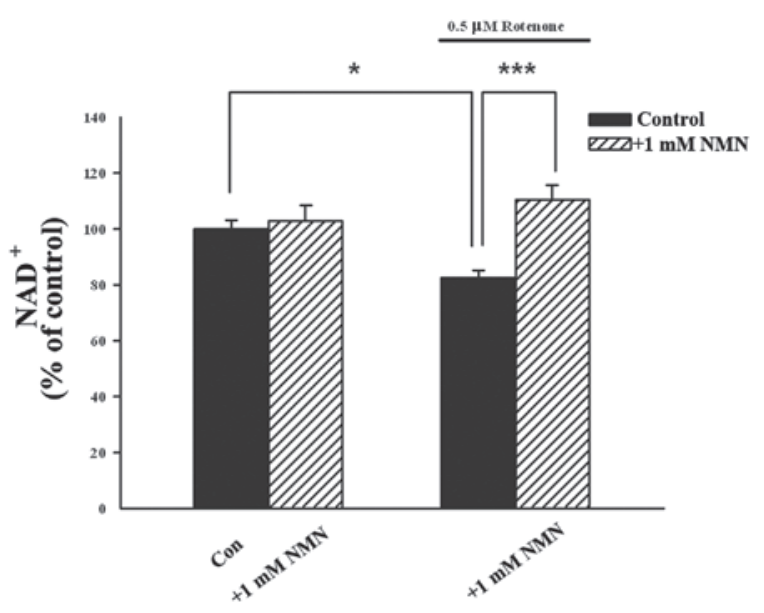

Figure 6. Nicotinamide mononucleotide (NMN) treatment attenuated the rotenone-induced reduction in the intracellular levels of nicotinamide adenine dinucleotide $\left(\mathrm{NAD}^{+}\right)$. PC12 cells were treated with $0.5 \mathrm{mM}$ rotenone, with or without co-treatment with $1 \mathrm{mM} \mathrm{NMN}$ for $24 \mathrm{~h}$. Subsequently, the intracellular levels of $\mathrm{NAD}^{+}$in the cells were determined. ${ }^{*}<0.05$; **** $\mathrm{P}<0.001$; Con, control.

A

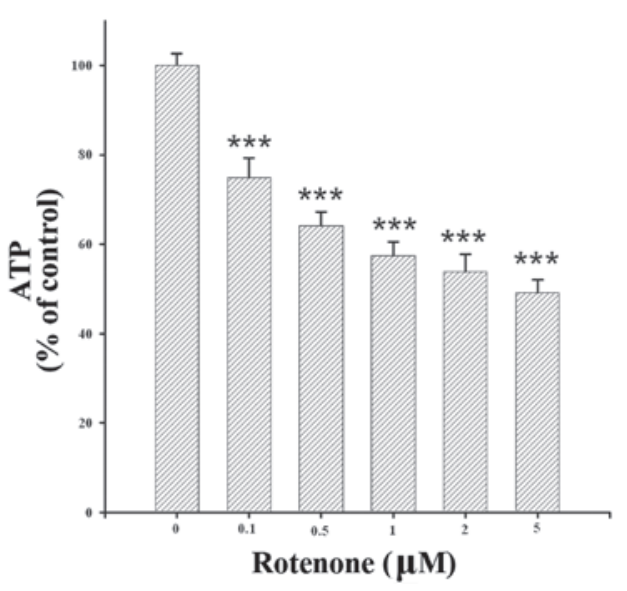

B

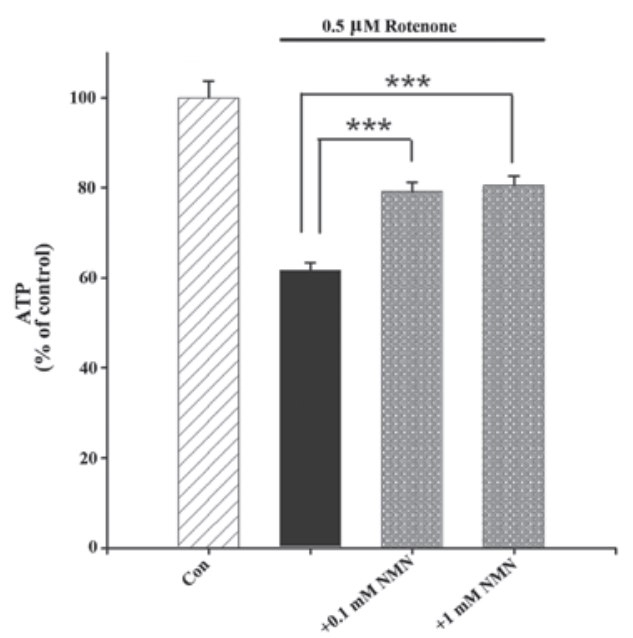

Figure 7. Nicotinamide mononucleotide (NMN) treatment attenuates the rotenone-induced reduction in intracellular levels of ATP in PC12 cells. (A) Rotenone concentration-dependently decreased the intracellular levels of ATP in PC12 cells, assessed by a luciferin/luciferase-based ATP assay. $\mathrm{PC} 12$ cells were treated with $0.5 \mathrm{mM}$ rotenone for $24 \mathrm{~h}$. (B) NMN treatment attenuated the rotenone-induced reduction in the intracellular levels of ATP in PC12 cells. PC12 cells were treated with $0.5 \mathrm{mM}$ rotenone, with or without co-treatment with NMN for $24 \mathrm{~h}$. Subsequently, the intracellular levels of ATP in the cells were assessed by a luciferin/luciferase-based ATP assay. ${ }_{* * * *} \mathrm{P}<0.001$ as indicated or vs. $0 \mathrm{mM}$ rotenone; Con, control. 
Apoptotic changes are major pathological transformations in PD and numerous other neurological diseases $(23,24)$. A compromise in energy metabolism may also play a significant role in the pathology of neurodegenerative disorders (9). The present study indicates that NMN treatment may be highly protective against not only apoptosis, but also energy compromise, in rotenone-treated PC12 cells.

These results suggest that NMN may become a promising drug for the treatment of PD and multiple other diseases in which apoptosis and energy compromise play significant pathological roles. Further in vivo studies of the effect of NMN on $\mathrm{PD}$ are required.

\section{Acknowledgements}

The authors would like to thank all participants of this study. This study was supported by the '973' National Program Grants for Basic Research No. 2007CB947900 (to SC), No. 2010CB945200 (to WY) and No. 2011CB504104 (to SC), the Key Discipline Program Grant of Shanghai Municipality No. S30202 (to SC), the Chinese National Science Foundation Grant No. 81171098 (to WY), the Pujiang Scholar Program Grant No. 09PJ1405900 (to WY) and the Research Grant of Shanghai Jiao Tong University for Interdisciplinary Research on Engineering and Physical Sciences (to WY).

\section{References}

1. Ying W: NAD ${ }^{+}$and $\mathrm{NADH}$ in cellular functions and cell death. Front Biosci 11: 3129-3148, 2006.

2. Ying $\mathrm{W}: \mathrm{NAD}^{+} / \mathrm{NADH}$ and $\mathrm{NADP}^{+} / \mathrm{NADPH}$ in cellular functions and cell death: regulation and biological consequences. Antioxid Redox Signal 10: 179-206, 2008.

3. Xia W, Wang Z, Wang Q, et al: Roles of NAD(+) / NADH and NADP(+) / NADPH in cell death. Curr Pharm Des 15: 12-19, 2009.

4. Alano CC, Ying W and Swanson RA: Poly(ADP-ribose) polymerase-1-mediated cell death in astrocytes requires $\mathrm{NAD}^{+}$ depletion and mitochondrial permeability transition. J Biol Chem 279: 18895-18902, 2004.

5. Ying W, Garnier P and Swanson RA: NAD ${ }^{+}$repletion prevents PARP-1-induced glycolytic blockade and cell death in cultured mouse astrocytes. Biochem Biophys Res Commun 308: 809-813, 2003.

6. Wang S, Xing Z, Vosler PS, et al: Cellular NAD replenishment confers marked neuroprotection against ischemic cell death: role of enhanced DNA repair. Stroke 39: 2587-2595, 2008.
7. Ying W, Wei G, Wang D, et al: Intranasal administration with $\mathrm{NAD}^{+}$profoundly decreases brain injury in a rat model of transient focal ischemia. Front Biosci 12: 2728-2734, 2007.

8. Fearnley JM and Lees AJ: Ageing and Parkinson's disease: substantia nigra regional selectivity. Brain 114: 2283-2301, 1991.

9. Beal MF: Mitochondria, oxidative damage, and inflammation in Parkinson's disease. Ann NY Acad Sci 991: 120-131, 2003.

10. Bredesen DE, Rao RV and Mehlen P: Cell death in the nervous system. Nature 443: 796-802, 2006.

11. Simola N, Pinna A and Fenu S: Pharmacological therapy of Parkinson's disease: current options and new avenues. Recent Pat CNS Drug Discov 5: 221-238, 2010.

12. Beal MF: Therapeutic approaches to mitochondrial dysfunction in Parkinson's disease. Parkinsonism Relat Disord 15 (Suppl 3): S189-S194, 2009.

13. Olanow CW: The pathogenesis of cell death in Parkinson's disease - 2007. Mov Disord 22 (Suppl 17): S335-S342, 2007.

14. Bové J, Prou D, Perier C and Przedborski S: Toxin-induced models of Parkinson's disease. NeuroRx 2: 484-494, 2005.

15. Ying W and Swanson RA: The poly(ADP-ribose) glycohydrolase inhibitor gallotannin blocks oxidative astrocyte death. Neuroreport 11: 1385-1388, 2000.

16. Ying W, Han SK, Miller JW and Swanson RA: Acidosis potentiates oxidative neuronal death by multiple mechanisms. J Neurochem 73: 1549-1556, 1999.

17. Roy MK, Takenaka M, Kobori M, Nakahara K, Isobe S and Tsushida T: Apoptosis, necrosis and cell proliferation-inhibition by cyclosporine A in U937 cells (a human monocytic cell line). Pharmacol Res 53: 293-302, 2006.

18. Ma Y, Chen H, Xia W and Ying W: Oxidative stress and PARP activation mediate the NADH-induced decrease in glioma cell survival. Int J Physiol Pathophysiol Pharmacol 3: 21-28, 2011.

19. Nie H, Chen H, Han J, et al: Silencing of SIRT2 induces cell death and a decrease in the intracellular ATP level of PC12 cells. Int J Physiol Pathophysiol Pharmacol 3: 65-70, 2011.

20. Zimmermann $M$ and Meyer N: Annexin V/7-AAD staining in keratinocytes. Methods Mol Biol 740: 57-63, 2011.

21. Alano CC, Garnier P, Ying W, Higashi Y, Kauppinen TM and Swanson RA: NAD ${ }^{+}$depletion is necessary and sufficient for poly(ADP-ribose) polymerase-1-mediated neuronal death. J Neurosci 30: 2967-2978, 2010.

22. Huang F, Vemuri MC and Schneider JS: Modulation of ATP levels alters the mode of hydrogen peroxide-induced cell death in primary cortical cultures: effects of putative neuroprotective agents. Brain Res 997: 79-88, 2004.

23. Schulz JB: Anti-apoptotic gene therapy in Parkinson's disease. J Neural Transm Suppl: 467-476, 2006.

24. Liou AK, Clark RS, Henshall DC, Yin XM and Chen J: To die or not to die for neurons in ischemia, traumatic brain injury and epilepsy: a review on the stress-activated signaling pathways and apoptotic pathways. Prog Neurobiol 69: 103-142, 2003. 